For researchers, the tables should:

- Help to promote collaboration between teams working on tropical and Mediterranean animal feeding.

- Help to identify areas of incomplete knowledge, thereby stimulating needed research.

\section{Methodology}

For each feed material or family of feed materials, we will use the following methodology:

- Research and identification of relevant databases and scientific literature

- Collection and summarizing quantitative and qualitative feed data

- Establishing representative and consistent vectors of chemical and nutritive values. For large families of feedstuffs, the methods of meta-analysis will be used.

- The project is managed through a collaborative website that is editable on-line by the contributors. A database contains the composition and nutritive data, equations and calculation processes.

\section{Current results}

- A database containing more than 2 million raw data has been established.

- Specifications have been drawn up for researching information and writing datasheets.

- Collaborations have begun with Gembloux Agrobiotech (Belgium), the Hassan II Institute (Morocco) and the University of Cordoba (Spain). Other institutions are interested in participating.

- A first batch of 100 datasheets will be produced by the end of 2010 .

\section{To be done}

- Establishing a formal methodology for calculating final table values

- Generation of approximately 200 datasheets per year for the next 3 years.

- Finding new partners in other countries in order to increase the breadth of both data collection and expertise

- Development of a website in partnership with the FAO. The website content will be maintained on a permanent basis to provide updated information to feed users.

\title{
Sub-saharan African feed database
}

\author{
Alan J. Duncan ${ }^{1}$, Bruno Gerard ${ }^{2}$ and Katrien Descheemaeker ${ }^{1,3}$ \\ ${ }^{1}$ International Livestock Research Institute, PO Box 5689, Addis Ababa, Ethiopia; ${ }^{2}$ CGIAR Systemwide Livestock Programme, ILRI, PO Box 5689, Addis Ababa, \\ Ethiopia; ${ }^{3}$ Current address : CSIRO, Ecosystem Sciences, BMP 2, Glen Osmond, SA 5064, Australia \\ Email : A.Duncan@cgiar.org
}

SSA Feeds is a web-based database of nutritional parameters for a range of feeds commonly fed to livestock in Sub-Saharan Africa. The database is reasonably comprehensive and provides advanced search functions as well as allowing descriptive statistics for sub-sets of data to be generated. The database is a useful resource for extension, development and research agents to design scientifically-based rations for meat, dairy and draught animals of small-scale African farmers. The database also contains useful nutritional data for development of nutritional components of farm household models such as lifesim (http://vs/p.org/models/).

\section{What is SSA Feeds?}

SSA Feeds is a database of nutritive values of feeds commonly fed to livestock in Sub-Saharan Africa. The database is freely available on the internet (http://vslp.org/ssafeed/) and is widely used. The database contains information on 459 livestock feed types from 14 countries in sub-Saharan Africa. The data is based on 14571 samples analysed over the years in the nutrition labs of the International Livestock Research Institute. An additional database provides nutritional data for Ethiopian feeds derived from the literature including references to source material.The database has strong search capabilities allowing searches by country, feed type, genus and species and also a user-friendly free search facility. The web-based interface also allows information from a range of feed samples to be summarised using descriptive statistics including mean values, standard deviation, minimum value and maximum value. Raw data can be exported to spreadsheets for further data analysis.

\section{What parameters?}

The database includes a comprehensive range of measures of nutritive value including the «Van Soest fibre» parameters, NDF and ADF as well as in vitro dry matter digestibility (IVDMD). Metabolizable energy values are estimated from IVDMD and net energy values for maintenance, gain and lactation are also calculated within the database. Crude protein values estimated as $6.25 \times$ Kjeldahl nitrogen concentrations are also presented. Finally a range of important trace element concentrations are available.

\section{Who is it useful for?}

The data presented in the database is designed to enable extension, development and research agents to design scientifically-based and best-cost rations for meat, dairy and draught animals of small-scale African farmers. The data will also be useful for research purposes, in particular for parameterizing nutritional components of farm household models such as lifesim (http://vslp.org/models/). The database fills a gap in global feed data since most other data sources neglect the feeds which predominate under Sub-Saharan African conditions. 